ESAIM: PROCEEDINGS, April 2009, Vol. 26, p. 24-44

H. Ammari, Editor

\title{
IMAGE PROCESSING BY TOPOLOGICAL ASYMPTOTIC ANALYSIS
}

\author{
Didier Auroux ${ }^{1}$ And Mohamed Masmoudi ${ }^{1}$
}

\begin{abstract}
The aim of this article is to recall the applications of the topological asymptotic expansion to many image processing problems. We briefly review the topological asymptotic analysis. A very natural application of this technique in image processing is the inpainting problem, which can be solved by identifying the optimal localization of the missing edges. A second natural application is then the image restoration or enhancement problem. The identification of the main edges of the image allows us to preserve them, and to smooth the image outside the edges. We present then an application to the regularized and unsupervised classification problem. If the conductivity outside the edges goes to infinity, the regularized image is piecewise constant and provides a natural solution to the segmentation problem. We also mention that all these problems are solved with a $\mathcal{O}(n \cdot \log (n))$ complexity. Finally, we present a hybrid scheme for contour detection and completion based on topological gradient and fast marching algorithms. We briefly illustrate all these applications with numerical results.
\end{abstract}

Résumé. Dans cet article, nous présentons l'application du gradient topologique à plusieurs problèmes classiques en traitement d'images. Après avoir brièvement introduit l'analyse asymptotique topologique, nous présentons son application naturelle au problème de l'inpainting, qui peut être résolu en identifiant la localisation optimale des contours manquants dans l'image. Une deuxième application naturelle est la restauration ou le débruitage d'images. L'identification des principaux contours permet de les préserver au cours du processus de restauration. Nous présentons ensuite une application à la classification régularisée et non supervisée. Lorsque la conductivité tend vers l'infini en dehors des contours, l'image régularisée devient constante par morceaux, et fournit une solution naturelle au problème de segmentation d'une image. Nous précisons ensuite la complexité de tous ces algorithmes, en $\mathcal{O}(n$. $\log (n)$. Enfin, nous présentons un algorithme hybride pour la détection et fermeture des contours d'une image, basé sur les algorithmes du gradient topologique et des chemins minimaux. Pour chacune des applications, nous illustrons l'algorithme avec quelques résultats numériques.

\section{INTRODUCTION}

The idea of topological asymptotic analysis is to measure the impact of a perturbation of the domain on a cost function. We only consider here the approach that has been introduced for topological optimization purpose, in which the goal is to identify an optimal shape and its complementary in a given domain $[38,39,42]$.

Topological shape optimization seems particularly well adapted to solve image processing problems (like classification, segmentation, enhancement, inpainting, ...), as they mainly consist of identifying a particular subdomain of the image: its edges.

At first sight, the main issue of topological shape analysis is the non-differentiability of the problem. To find the optimal domain is indeed equivalent to identify its characteristic function. Several classical approaches have been developed to make this problem differentiable. We can cite here the relaxation technique, which allows

\footnotetext{
1 Institut de Mathématiques de Toulouse, Université Paul Sabatier Toulouse 3, 31062 Toulouse cedex 9, France; e-mail: auroux@math.univ-toulouse.fr
}

(c) EDP Sciences, SMAI 2009 Article published by EDP Sciences and available at http://www.edpsciences.org/proc or http://dx.doi.org/10.1051/proc/2009003 
the characteristic function to take all possible values in the interval $[0 ; 1]$, and the level set approach where the characteristic function is replaced by a regular level set function which is positive inside the optimal domain and negative outside [4-6,21,42,52].

The idea of topological asymptotic analysis is to switch the characteristic function from one to zero (or from zero to one) in a (infinitely) small area. Thus, the variation of the cost function is small when we switch a very small part from the subdomain to its complementary. The topological asymptotic expansion provides this variation, and allows one to derive a topological gradient of the cost function $[38,42,52,53]$.

In this article, we first present the basic tools of topological asymptotic analysis, and we then study several applications to image processing problems: inpainting (where the goal is to fill a hidden part of an image), restoration and enhancement, classification, and segmentation. Then, we present a very efficient way to speed up all the algorithms introduced in this article, based on discrete cosine transforms and an appropriate preconditioning. Finally, we present a coupled approach combining the topological gradient and the minimal path technique in order to improve the edge detection, and to avoid non-connex contours.

\section{TOPOlOGiCAL ASYMPtotic ANALYSis}

\subsection{Presentation of the method}

Let $\Omega$ be a regular open bounded domain of $\mathbb{R}^{2}$ (or $\mathbb{R}^{3}$ ). Let us consider a Partial Differential Equation (PDE) problem defined in $\Omega$, written in its variational formulation:

$$
\text { find } u \in \mathcal{V} \text { such that } a(u, w)=l(w), \forall w \in \mathcal{V}
$$

where $\mathcal{V}$ is a Hilbert space on $\Omega$, usually $H^{1}(\Omega), a$ is a bilinear continuous and coercive form defined on $\mathcal{V}$, and $l$ is a linear continuous form on $\mathcal{V}$. We finally consider a cost function $J(\Omega, u)$ to be minimized, where $u$ is the solution of equation (1).

We now consider a small perturbation of the domain, e.g. by the insertion of a crack $\sigma_{\rho}=x_{0}+\rho \sigma(n)$, where $x_{0} \in \Omega$ represents the point where the crack is inserted, $\sigma(n)$ is a straight crack containing the origin of the domain, and $n$ is a unit vector normal to the crack. Finally, $\rho>0$ represents the size of the perturbation, assumed to be small. Let $\Omega_{\rho}=\Omega \backslash \sigma_{\rho}$ be the perturbed domain. We can consider the same PDE problem as before, but on the perturbed domain:

$$
\text { find } u_{\rho} \in \mathcal{V}_{\rho} \text { such that } a_{\rho}\left(u_{\rho}, w\right)=l_{\rho}(w), \forall w \in \mathcal{V}_{\rho}
$$

where $\mathcal{V}_{\rho}, a_{\rho}$ and $l_{\rho}$ represent the restriction of the Hilbert space $\mathcal{V}$ to $\Omega_{\rho}$, and the perturbed bilinear and linear forms respectively.

We can rewrite the cost function $J$ as a function of $\rho$ by considering the following map:

$$
j: \rho \mapsto \Omega_{\rho} \mapsto u_{\rho} \text { solution of }(2) \mapsto j(\rho):=J\left(\Omega_{\rho}, u_{\rho}\right)
$$

The topological sensitivity theory provides an asymptotic expansion of $j$ when $\rho$ tends to zero. It takes the general form:

$$
j(\rho)-j(0)=f(\rho) G\left(x_{0}\right)+o(f(\rho)),
$$

where $f(\rho)$ is an explicit positive function going to zero with $\rho$, and $G\left(x_{0}\right)$ is called the topological gradient at point $x_{0}[42]$.

Then to minimize the criterion $j$, one has to insert small holes (or cracks) at points where the topological gradient $G$ is the most negative, in order to make the cost function $j$ decrease quickly (see the asymptotic expansion (4)). 


\subsection{Main result}

In the following, we will consider several times this main result [7]:

Theorem 1.1. If there exists a linear form $L_{\rho}$ defined on $\mathcal{V}_{\rho}$, a function $f: \mathbb{R}^{+} \rightarrow \mathbb{R}^{+}$, and four real numbers $\delta J_{1}, \delta J_{2}, \delta a$ and $\delta l$ such that

- $\lim _{\rho \rightarrow 0} f(\rho)=0$,

- $J\left(\Omega_{\rho}, u_{\rho}\right)-J\left(\Omega_{\rho}, u_{0}\right)=L_{\rho}\left(u_{\rho}-u_{0}\right)+f(\rho) \delta J_{1}+o(f(\rho))$,

- $J\left(\Omega_{\rho}, u_{0}\right)-J\left(\Omega, u_{0}\right)=f(\rho) \delta J_{2}+o(f(\rho))$,

- $\left(a_{\rho}-a_{0}\right)\left(u_{0}, p_{\rho}\right)=f(\rho) \delta a+o(f(\rho))$,

- $\left(l_{\rho}-l_{0}\right)\left(p_{\rho}\right)=f(\rho) \delta l+o(f(\rho))$,

where the adjoint state $p_{\rho}$ is the solution of the adjoint equation

$$
a_{\rho}\left(w, p_{\rho}\right)=-L_{\rho}(w), \forall w \in \mathcal{V}_{\rho},
$$

and $u_{\rho}$ is the solution of the direct equation (2), then the cost function $j$ has the asymptotic expansion (4), where the topological gradient $G(x)$ is given by

$$
G(x)=\delta J_{1}+\delta J_{2}+\delta a-\delta l
$$

\section{INPAINTING}

In this section, we present an application of the topological asymptotic analysis to the inpainting problem. The goal of inpainting is to fill a hidden part of an image. In other words, if we denote by $\Omega$ the original image and $\omega$ the hidden part of the image, the goal is to recover the hidden part $\omega$ from the known part of the image $\Omega \backslash \omega$. There are many applications, for instance removing some spots on a badly preserved movie or image, or deleting encrusted logos and images on television programs, ...

This problem has been widely studied. Several methods have been considered: learning approaches (neural networks, radial basis functions, support vector machine, ... ), in which the learning data is taken in $\Omega \backslash \omega$, and then the approximate function is evaluated in $\omega[58,59]$; minimization of an energy cost function in $\omega$ based on a total variation norm $[28,29]$; morphological analysis for the reconstruction of both cartoon and texture [36]; ...

In order to study the inpainting problem, we first consider a crack localization method. Crack detection allows us to identify the edges of the hidden part of the image, and the inpainting problem can then be easily solved. We will consider the classical thermal diffusion technique $[27,45,48,55,56]$ and improve it by modeling the edges by cracks. These cracks are supposed to be highly insulating and to allow the temperature to jump across edges. As both the Dirichlet and Neumann conditions are known on the boundary of the hidden subset, we can define a criterion measuring the discrepancy between the solutions of a Dirichlet and a Neumann problem respectively [40]. This problem is similar to the inverse conductivity problem, also known as the Calderón problem [26], which consists of identifying the coefficients of a partial differential equation from the knowledge of the Dirichlet to Neumann operator. Only two measurements are needed to recover several simple cracks $[2,3,18]$. From the numerical point of view, several methods $[8,19,20,25,37,49,50]$ have been proposed, but the topological gradient approach seems to be the most efficient method for crack localization. The minimization of the criterion allows us to identify the main edges inside the hidden part of the image. The image is finally filled between the edges thanks to the Laplace operator.

This section summarizes the work introduced in $[15,16]$. We also refer to these references for the results of many numerical experiments.

\subsection{Crack localization problem}

Let $\Omega$ be a bounded open set of $\mathbb{R}^{2}$. We assume in this section that $\Omega$ contains a perfectly insulating crack $\sigma^{*}$. We impose a flux $\phi \in H^{-1 / 2}(\Gamma)$ on the boundary $\Gamma$ of $\Omega$, and we want to find $\sigma \subset \Omega$ such that the solution 
$u \in H^{1}(\Omega \backslash \sigma)$ of

$$
\begin{cases}\Delta u=0 & \text { in } \Omega \backslash \sigma, \\ \partial_{n} u=\phi & \text { on } \Gamma, \\ \partial_{n} u=0 & \text { on } \sigma,\end{cases}
$$

satisfies $\left.u\right|_{\Gamma}=T$, where $T \in H^{1 / 2}(\Gamma)$ is a given function. We also assume some compatibility conditions in order to have a well-posed direct problem.

A topological gradient approach has been introduced in [7], and consists of defining a Dirichlet and a Neumann problem, as we have an over-determination in the boundary conditions:

$$
\begin{gathered}
u_{D} \in H^{1}(\Omega \backslash \sigma) \text { such that }\left\{\begin{array}{l}
\Delta u_{D}=0 \text { in } \Omega \backslash \sigma, \\
u_{D}=T \text { on } \Gamma, \\
\partial_{n} u_{D}=0 \text { on } \sigma,
\end{array}\right. \\
u_{N} \in H^{1}(\Omega \backslash \sigma) \text { such that }\left\{\begin{array}{l}
\Delta u_{N}=0 \text { in } \Omega \backslash \sigma, \\
\partial_{n} u_{N}=\phi \text { on } \Gamma, \\
\partial_{n} u_{N}=0 \text { on } \sigma .
\end{array}\right.
\end{gathered}
$$

It is clear that for the actual crack $\sigma^{*}$, the two solution $u_{D}$ and $u_{N}$ are equal. The idea is then to consider and minimize the following cost function

$$
J(\sigma)=\frac{1}{2}\left\|u_{D}-u_{N}\right\|_{L^{2}(\Omega)}^{2}
$$

The topological asymptotic expansion of this cost function is detailed in [7].

\subsection{Dirichlet and Neumann formulations for the inpainting problem}

In our approach, we now denote by $\Omega$ the image and $\Gamma$ its boundary, $\omega \subset \Omega$ the missing part of the image and $\gamma$ its boundary. Let $v$ be the image that we want to restore. We assume that $v$ is known in $\Omega \backslash \omega$, and unknown in $\omega$.

The idea is to adapt the crack localization method to inpainting: crack detection first allows us to identify the cracks (or edges) $\sigma$ of the hidden part $\omega$ of the image, and then we will impose that the Laplacian of the restored image is equal to zero in $\omega \backslash \sigma$. For a given crack $\sigma \subset \omega$, as $v$ (Dirichlet condition) and $\partial_{n} v$ (Neumann condition) are known on the boundary $\gamma$ of $\omega$, we can solve two different problems inside $\omega$.

For a given crack $\sigma$, we denote by $u_{D} \in H^{1}(\Omega \backslash \sigma)$ the solution of the following Dirichlet problem:

$$
\left\{\begin{array}{l}
\Delta u_{D}=0 \quad \text { in } \omega \backslash \sigma \\
u_{D}=v \quad \text { on } \gamma \\
\partial_{n} u_{D}=0 \quad \text { on } \sigma \\
u_{D}=v \quad \text { in } \Omega \backslash \omega .
\end{array}\right.
$$

Outside $\omega$, the solution is equal to the original image, and inside $\omega$, we use equation (8).

In the same way, if we assume $v$ to be enough regular, we can consider the solution $u_{N} \in H^{1}(\Omega \backslash \sigma)$ of the following Neumann problem:

$$
\left\{\begin{array}{l}
\Delta u_{N}=0 \quad \text { in } \omega \backslash \sigma \\
\partial_{n} u_{N}=\partial_{n} v \quad \text { on } \gamma \\
\partial_{n} u_{N}=0 \text { on } \sigma \\
u_{N}=v \text { in } \Omega \backslash \omega
\end{array}\right.
$$


Note that from the numerical point of view, it is much more easy to solve an approximated Neumann problem:

$$
\left\{\begin{array}{l}
\Delta u_{N}=0 \text { in } \omega \backslash \sigma, \\
\partial_{n} u_{N}=\partial_{n} v \text { on } \gamma, \\
\partial_{n} u_{N}=0 \text { on } \sigma, \\
-\alpha \Delta u_{N}+u_{N}=v \quad \text { in } \Omega \backslash \omega,
\end{array}\right.
$$

where $\alpha$ is a small positive number.

\subsection{Asymptotic expansion}

The cost function remains unchanged, and is still defined by (10), as the idea is to find some cracks $\sigma \subset \omega$ that minimize the difference between the two solutions $u_{N}$ and $u_{D}$. We assume that the crack $\sigma$ is equal to $x+\rho \bar{\sigma}$, where $x$ is the point of insertion of the crack, $\rho$ is the size of the inserted crack (assumed to be small), and $\bar{\sigma}$ is a reference crack, of unit normal vector $n$. Then, we can rewrite the cost function $J$ defined by equation (10) as a function $j(\rho)$ of $\rho$. The asymptotic expansion is then the following:

$$
j(\rho)-j(0)=f(\rho) g(x, n)+o(f(\rho)),
$$

where the topological gradient $g$ is defined by

$$
g(x, n)=-\left[\left(\nabla u_{D}(x) \cdot n\right)\left(\nabla p_{D}(x) \cdot n\right)+\left(\nabla u_{N}(x) \cdot n\right)\left(\nabla p_{N}(x) \cdot n\right)\right],
$$

where $u_{D}$ and $u_{N}$ are the solutions of (11) and (12) respectively, but without any inserted crack $(\sigma=\emptyset)$. Also, $p_{D}$ and $p_{N}$ are the corresponding adjoint states, respectively solutions in $H^{1}(\Omega)$ of the following equations:

$$
\begin{aligned}
& \left\{\begin{array}{l}
p_{D}=0 \text { in } \Omega \backslash \omega, \\
p_{D}=0 \text { on } \gamma, \\
-\Delta p_{D}=-\left(u_{D}-u_{N}\right) \quad \text { in } \omega,
\end{array}\right. \\
& \left\{\begin{array}{l}
p_{N}=0 \text { in } \Omega \backslash \omega, \\
\partial_{n} p_{N}=0 \text { on } \gamma, \\
-\Delta p_{N}=+\left(u_{D}-u_{N}\right) \text { in } \omega .
\end{array}\right.
\end{aligned}
$$

The topological gradient defined by equation (15) can be rewritten in the following way:

$$
g(x, n)=n^{T} M(x) n,
$$

where $M(x)$ is the $2 \times 2$ (resp. $3 \times 3$ in the case of $3 \mathrm{D}$ images, or movies) symmetric matrix defined by

$$
M(x)=-\operatorname{sym}\left(\nabla u_{D}(x) \otimes \nabla p_{D}(x)+\nabla u_{N}(x) \otimes \nabla p_{N}(x)\right) .
$$

From this equation, we can deduce that the minimum of $g(x, n)$ is reached when $n$ is the eigenvector associated to the lowest eigenvalue $\lambda_{\min }(M(x))$ of $M(x)$.

\subsection{Algorithm}

The inpainting algorithm is then the following:

- Calculation of $u_{D}$ and $u_{N}$, solutions of the direct problems (11) and (12) respectively, without any inserted crack (unperturbed problem: $\sigma=\emptyset$ ).

- Calculation of $p_{D}$ and $p_{N}$ the two corresponding adjoint states, respectively solutions of equations (16) and (17).

- Computation of the matrix $M(x)$ defined by equation (19). 

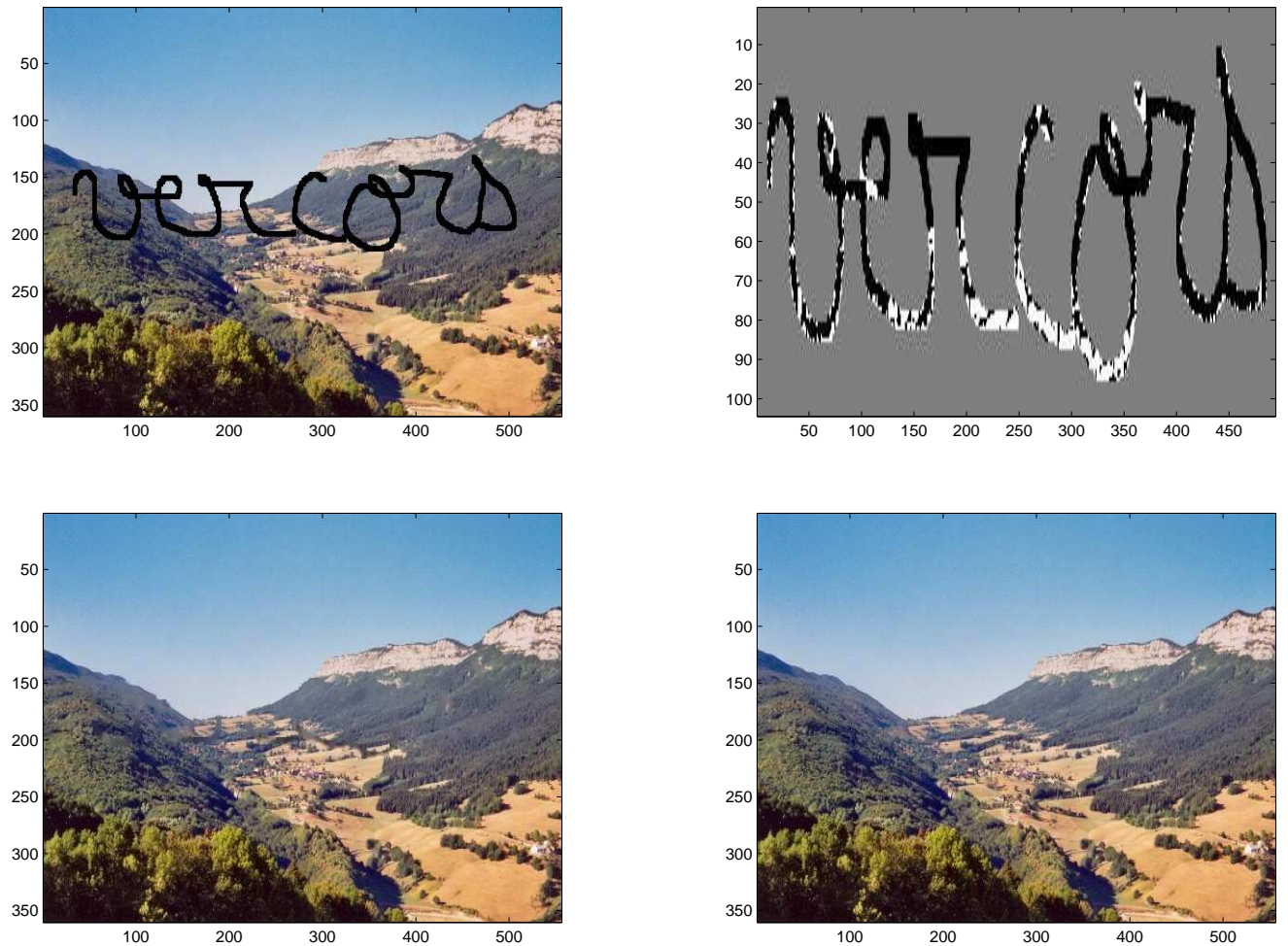

Figure 1. Top: Noisy image (left); Identified missing edges (in white) by the topological gradient algorithm (right). Bottom: Inpainted (or reconstructed) image (left); Original image, for comparison (right).

- Localization of the cracks: define

$$
\sigma=\left\{x \in \omega ; \lambda_{\min }(M(x))<\delta<0\right\}
$$

where $\delta$ is a negative threshold.

- Calculation of the solution of the Neumann problem (12) perturbed by the insertion of $\sigma$.

This image is then equal to the original image in $\Omega \backslash \omega$, and it has been reconstructed in $\omega$.

Figure 1 illustrates this algorithm. We refer to [15] for more numerical results.

\subsection{Remarks}

From the numerical point of view, cracks are modeled by a very small conductivity instead of considering real holes in the domain. The previous algorithm has a complexity of $\mathcal{O}(n \cdot \log (n))$, where $n$ is the size of the image, i.e. the number of pixels, as explained in section 6 .

The main advantage of this algorithm is that the reconstruction is done in only one iteration of the topological gradient algorithm, which consists of 5 resolutions of a PDE (the two direct and two adjoint unperturbed problems, and then one direct perturbed problem) in the domain $\Omega$ representing the image. Several numerical results are presented in [15] and show the quality and efficiency of the reconstruction.

The only control parameter of this method is the negative threshold: below a given value, the pixels are considered as being part of the edge set, whereas it is not the case beyond the threshold. The reconstructed 
image is provided by the resolution of the direct perturbed problem (12), and the quality of the image relies on the connexity of the identified edges. If a given identified edge is not connex, the Laplacian indeed produces a blurred zone. Then, the threshold is usually set such that the main identified edges are connex. Of course, it may lead to the wrong identification of edges. But the various numerical experiments have shown that the threshold can be fixed to an a priori value, as the optimal threshold is almost independent of the images.

Another solution to this problem is presented in section 7.

\section{Restoration}

In this section, we consider the restoration problem, with the aim of restoring noisy images. The main idea is to use the topological gradient for detecting the edges of the noisy image in order to preserve them during the restoration process.

This method is based on thermal diffusion, like many other variational methods. In order to avoid blurring effects, several nonlinear isotropic and anisotropic methods have been introduced, some of them relying on the minimization of the total variation $[10,27,41,45,55,56]$. We should mention that some non variational approaches also exist, mainly statistical methods [35].

This section summarizes the work presented in $[14,17]$. We also refer to these references for the results of numerical experiments.

\subsection{Variational formulation}

Let $\Omega \subset \mathbb{R}^{2}$ be an open bounded domain, and $v \in L^{2}(\Omega)$ be the noisy image. The enhancement of $v$ is based on the resolution of the following problem:

$$
\text { find } u \in H^{1}(\Omega) \text { such that } \begin{cases}-d i v(c \nabla u)+u=v & \text { in } \Omega, \\ \partial_{n} u=0 & \text { on } \quad \partial \Omega,\end{cases}
$$

where $n$ is the outward unit normal to $\partial \Omega$, and $c$ is the conductivity, to be defined in the following. Several choices can be made for the conductivity, mainly $c$ equal to a constant value (linear diffusion method: it is fast, but it blurs important structures), or $c$ defined by a nonlinear function of $\nabla u$ (nonlinear diffusion method, edge-preserving $[10,56])$. In the topological gradient approach, $c$ takes only two values: a constant value $c_{0}$ (close to 1 ) in the smooth part of the image, and a very small values $\varepsilon$ (close to 0 ) on the edges or cracks in order to preserve them.

Setting $c=0$ on a part of the image is equivalent to perturbing the domain by the insertion of cracks. For a given point $x_{0} \in \Omega$ and for a given small parameter $\rho>0$, we consider $\Omega_{\rho}=\Omega \backslash \sigma_{\rho}$ the perturbed domain by the insertion of a crack $\sigma_{\rho}=x_{0}+\rho \sigma(n)$, where $\sigma(n)$ is a straight crack and $n$ is a unit vector normal to the crack. The variational formulation of the perturbed problem is the following:

$$
\text { find } u_{\rho} \in H^{1}\left(\Omega_{\rho}\right) \text { such that } a_{\rho}\left(u_{\rho}, w\right)=l_{\rho}(w), \forall w \in H^{1}\left(\Omega_{\rho}\right) \text {, }
$$

where $a_{\rho}\left(\right.$ resp. $\left.l_{\rho}\right)$ is the following bilinear (resp. linear) form defined on $H^{1}\left(\Omega_{\rho}\right)$ (resp. $L^{2}\left(\Omega_{\rho}\right)$ ) by

$$
a_{\rho}(u, w)=\int_{\Omega_{\rho}}(c \nabla u \nabla w+u w) d x, \quad l_{\rho}(w)=\int_{\Omega_{\rho}} v w d x
$$

Edge detection if equivalent to looking for a subdomain of $\Omega$ where the energy is small. So our goal is to minimize the energy norm outside edges:

$$
j(\rho)=J\left(\Omega_{\rho}, u_{\rho}\right)=\int_{\Omega_{\rho}}\left\|\nabla u_{\rho}\right\|^{2} .
$$




\subsection{Topological gradient}

From theorem 1.1, we can derive the following asymptotic expansion of the cost function (24):

$$
j(\rho)-j(0)=\rho^{2} G\left(x_{0}, n\right)+o\left(\rho^{2}\right),
$$

where

$$
G\left(x_{0}, n\right)=-\pi c\left(\nabla u_{0}\left(x_{0}\right) \cdot n\right)\left(\nabla p_{0}\left(x_{0}\right) \cdot n\right)-\pi\left|\nabla u_{0}\left(x_{0}\right) \cdot n\right|^{2},
$$

where $p_{0}$ is the solution of the unperturbed adjoint problem:

$$
\begin{cases}-\operatorname{div}\left(c \nabla p_{0}\right)+p_{0}=-\partial_{u} J\left(\Omega, u_{0}\right) & \text { in } \quad \Omega, \\ \partial_{n} p_{0}=0 & \text { on } \quad \partial \Omega .\end{cases}
$$

As previously seen, the topological gradient can be rewritten: $G(x, n)=\langle M(x) n, n\rangle$, where $M(x)$ is the following $2 \times 2$ symmetric matrix:

$$
M(x)=-\pi c \frac{\nabla u_{0}(x) \nabla p_{0}(x)^{T}+\nabla p_{0}(x) \nabla u_{0}(x)^{T}}{2}-\pi \nabla u_{0}(x) \nabla u_{0}(x)^{T} .
$$

\subsection{Algorithm}

Our algorithm consists of inserting small heterogeneities (or cracks) in regions where the topological gradient is smaller than a given threshold. There regions are the edges of the image. The algorithm is as follows:

- Initialization: $c=c_{0}$ (constant value everywhere).

- Calculation of $u_{0}$ and $p_{0}$, respectively solutions of the direct (21) and adjoint (27) unperturbed problems.

- Computation of the $2 \times 2$ matrix $M(x)$ defined by (28), and of its lowest eigenvalue $\lambda_{\min }(M(x))$ at each point of the domain.

- Set the new conductivity:

$$
c_{1}=\left\{\begin{array}{l}
\varepsilon \text { if } x \in \Omega \text { is such that } \lambda_{\min }(M(x))<\alpha<0 \\
c_{0} \text { elsewhere }
\end{array}\right.
$$

where $\varepsilon>0$ is assumed to be small, and $\alpha$ is a negative threshold.

- Calculation of $u_{1}$, the solution of the perturbed direct problem (21) using $c=c_{1}$.

The image $u_{1}$ is the restored image.

Figure 2 illustrates this algorithm. We refer to $[14,17]$ for more numerical results.

\subsection{Remarks}

From the numerical point of view, it is more convenient to simulate the cracks by a small value of $c$ instead of considering topological perturbations of $\Omega$. The resolution of problem (21) with $c=c_{1}$ is an approximation of the resolution of the perturbed problem (22), becoming more precise as $\varepsilon$ goes to 0 .

As in the previous section (inpainting problems), our algorithm is extremely efficient as it requires only 3 resolutions of a partial differential equation in $\Omega$ : the direct and adjoint original problems, and then the direct perturbed problem. And the complexity of this algorithm is still $\mathcal{O}(n \cdot \log (n))$ (see section 6$)$.

As shown in [17], the quality of the numerical results is very good. Once again, the algorithm relies on a thresholding of the topological gradient in order to define the edge set. Contrary to inpainting problems, the connexity of the edges is not crucial since it does not change significantly the quality of the restored image. However, section 7 presents a way to identify connex edges, with fewer badly identified edges. 

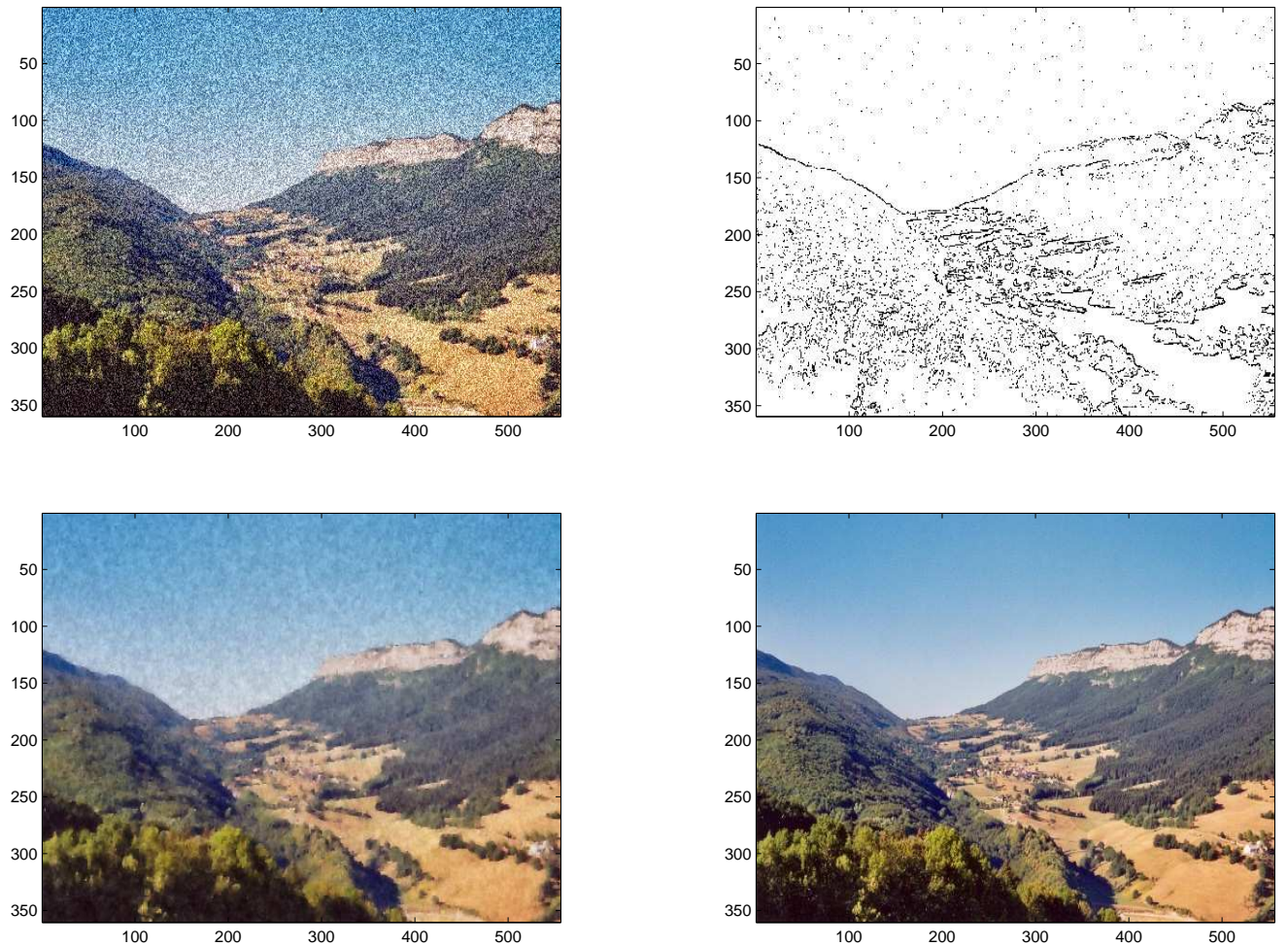

Figure 2. Top: Noisy image (left); Identified edges by the topological gradient algorithm (right). Bottom: Restored image (left); Original image, for comparison (right).

\subsection{Extension to color images}

In this section, we adapt the topological gradient approach to color images. Color images can be represented or modeled in various ways, for instance the RGB (Red-Green-Blue) space in which images are viewed as functions from $\Omega$ to $\mathbb{R}^{3}$ instead of $\mathbb{R}$. A first approach consists of decoupling the three channels, and in solving direct and adjoint problems for each channel. But it is also possible to consider directly the vectorial minimization problem, involving the resolution of vectorial problems. The topological asymptotic expansion is still given by equations (25-26) and (28), where all functions are vectorial, i.e. the topological gradient is the sum on all channels of the corresponding expressions for each channel [14].

Another approach has also been studied in [14], in which we use a different norm for coupling the different channels. In order to identify the local variations of the color image, Di Zenzo defines a multi-spectral tensor associated to the image vector field [33]:

$$
T=\left(\begin{array}{cc}
t_{11} & t_{12} \\
t_{21} & t_{22}
\end{array}\right), \quad t_{i j}=\sum_{k=1}^{3} \frac{\partial u^{k}}{\partial x_{i}} \frac{\partial u^{k}}{\partial x_{j}}, \quad 1 \leq i, j \leq 2,
$$

in the case of bidimensional images. This tensor describes the first order differential structure of the image, and the Di Zenzo gradient is given by the square root of the largest eigenvalue of the structure tensor:

$$
\|\nabla u\|_{D Z}=\frac{1}{\sqrt{2}}\left[t_{11}+t_{22}+\sqrt{\left(t_{11}-t_{22}\right)^{2}+4 t_{12}^{2}}\right]^{\frac{1}{2}} .
$$


It is possible to rewrite this gradient in a different way with the following function:

$$
H(\nabla u)=\frac{1+\sqrt{1-4 f(\nabla u)}}{2},
$$

where

$$
\begin{gathered}
f(\nabla u)=\frac{\operatorname{det}^{2}\left(\nabla u^{1}, \nabla u^{2}\right)+\operatorname{det}^{2}\left(\nabla u^{1}, \nabla u^{3}\right)+\operatorname{det}^{2}\left(\nabla u^{2}, \nabla u^{3}\right)}{\left(\left|\nabla u^{1}\right|^{2}+\left|\nabla u^{2}\right|^{2}+\left|\nabla u^{3}\right|^{2}\right)^{2}}, \\
\operatorname{det}^{2}\left(\nabla u^{s}, \nabla u^{t}\right)=\left(\frac{\partial u^{s}}{\partial x_{1}} \frac{\partial u^{t}}{\partial x_{2}}-\frac{\partial u^{t}}{\partial x_{1}} \frac{\partial u^{s}}{\partial x_{2}}\right)^{2} .
\end{gathered}
$$

Then, we can derive the asymptotic expansion of the cost function defined by equation (24) in which the norm is the Di Zenzo norm (31):

$$
G\left(x_{0}, n\right)=\sum_{k=1}^{3}\left[-\pi c\left(\nabla u_{0}^{k}\left(x_{0}\right) \cdot n\right)\left(\nabla v_{0}^{k}\left(x_{0}\right) \cdot n\right)-\pi H\left(\nabla u_{0}\left(x_{0}\right)\right)\left|\nabla u_{0}^{k}\left(x_{0}\right) \cdot n\right|^{2}\right]
$$

with our standard notations.

In [14], we show that this approach has the same computational cost as the vectorial approach (in which the different channels are decoupled), while it improves the edge detection, and hence it produces a better restored image, more precise on the edges of the image.

\section{Classification}

In this section, we now focus on the regularized and unsupervised image classification problem.

Inspired by the work presented in $[10,48]$, in which the authors propose a classification model coupled with a restoration process, we adapt here our approach based on the topological asymptotic analysis.

This section summarizes the work presented in $[13,17]$. We refer to these references for the numerical results.

\subsection{Introduction to the classification problem}

Let $v$ be the original image defined on an open set $\Omega$ of $\mathbb{R}^{2}$, and let $C_{i}, 1 \leq i \leq n$, be $n$ classes (i.e. grey or color levels). We first assume that thesse classes are predefined. The goal of image classification is to find a partition of $\Omega$ in subsets $\left\{\Omega_{i}\right\}_{i=1 \ldots n}$, such that $v$ is close to $C_{i}$ in $\Omega_{i}$.

A variational approach can be defined: it consists of a cost function measuring the difference between the original image and the classified image:

$$
J\left(\left(\Omega_{i}\right)_{i=1 \ldots n}\right)=\sum_{i=1}^{n} \int_{\Omega_{i}}\left(v(x)-C_{i}\right)^{2} d x+\alpha \sum_{i \neq j}\left|\Gamma_{i j}\right|,
$$

where $\Gamma_{i j}$ represents the interface $\Omega_{i} \cap \Omega_{j}$ between two subsets.

The main difficulty of this approach is that the unknowns are sets, and not variables. This is why the topological asymptotic analysis seems to be appropriate for solving this problem. The topological gradient and the corresponding numerical results are presented in [13].

\subsection{Restoration and classification coupling}

Another solution consists of coupling classification with restoration, and to adapt the approach introduced in section 3. The idea is to first consider an iteration of the topological asymptotic analysis for the image restoration problem in order to smooth the image, and then to classify this smooth image without any regularization. If we remove the regularization term from equation (36), which leads to the unregularized classification problem, 

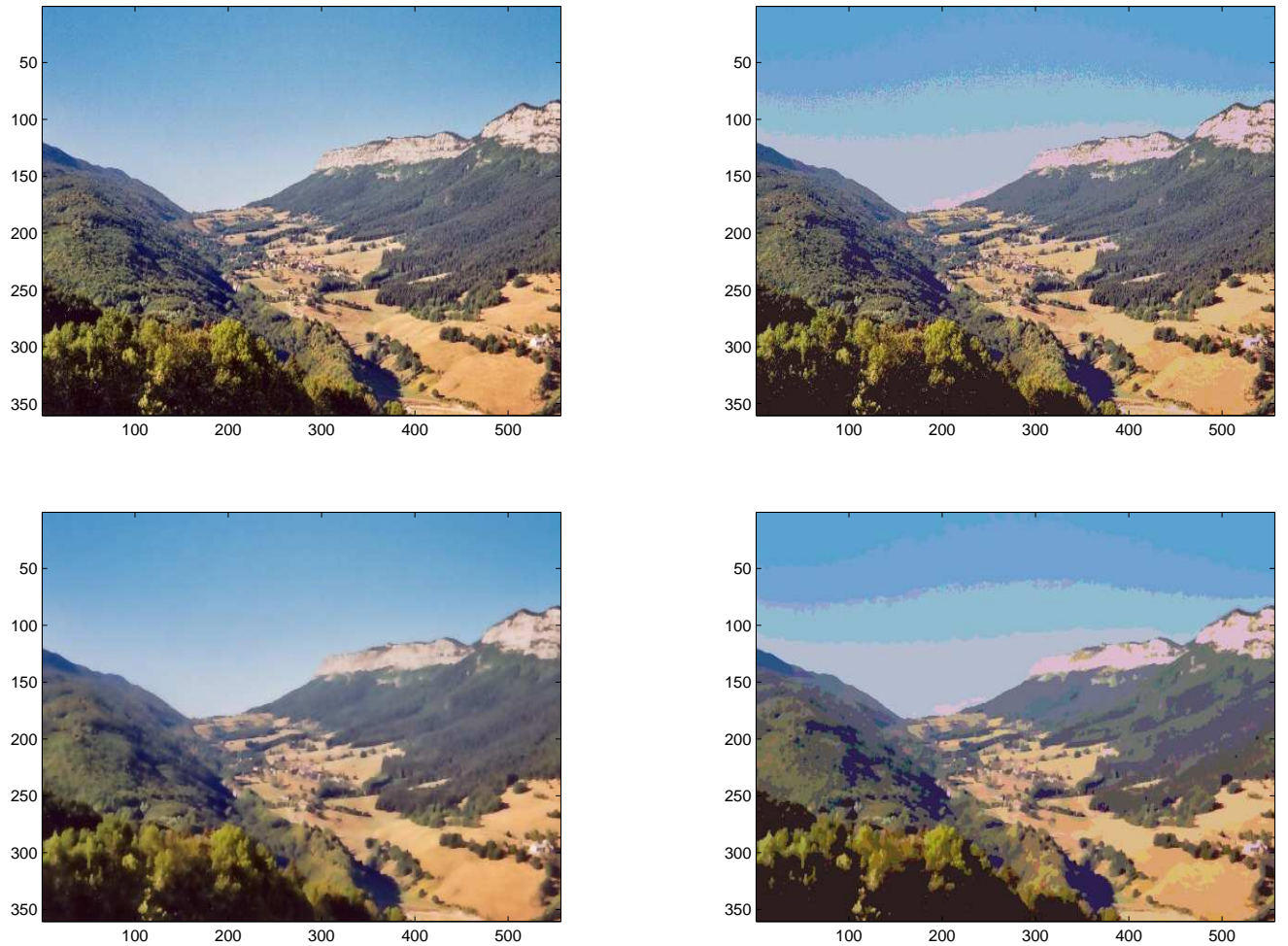

Figure 3. Top: Original image (left); Unregularized classified image (with an optimal choice of classes) (right). Bottom: Smooth image provided by the topological gradient algorithm (left); Classification of this smooth image (right).

then the optimal subset $\Omega_{i}$ is the set of pixels that are closer to $C_{i}$ than to any other $C_{j}$. In other words, each pixel is assigned to the subset corresponding to its closest class.

In the perturbed problem (29), instead of setting $c=0$ (or $c=\varepsilon$ from the numerical point of view) on the edge set and $c=c_{0}$ elsewhere, we set

$$
c_{1}=\left\{\begin{array}{l}
\varepsilon \text { on the edge set } \\
\frac{c_{0}}{\varepsilon} \text { elsewhere }
\end{array}\right.
$$

The algorithm is then the following:

- Application of the restoration algorithm defined in section 3 , with $c_{1}$ defined by (37) instead of (29).

- Unregularized classification of the image $u_{1}$, using for example the closest class algorithm (in which each pixel is assigned to the subset corresponding to its closest class).

Figure 3 illustrates this algorithm. We refer to $[13,17]$ for more numerical results.

As previously seen, the complexity of this algorithm is $\mathcal{O}(n \cdot \log (n))$, and the various numerical results presented in [13] show the relative efficiency of these approaches. Moreover, it is possible to regularize more or less the image by choosing different values of $c_{1}$, and it allows us to also obtain good results on noisy images. 


\subsection{Extension to unsupervised classification}

If the number $n$ of classes is given, but not their values $C_{i}$, it is possible to determine them in an optimal way. This classification problem can be defined as

$$
\min _{\left(\Omega_{i}\right),\left(C_{i}\right)} j\left(\left(\Omega_{i}\right),\left(C_{i}\right)\right)=\sum_{i=1}^{n} \int_{\Omega_{i}}\left|v(x)-C_{i}\right|^{2} d x+\alpha \sum_{i \neq j}\left|\Gamma_{i j}\right| .
$$

The idea is to minimize the cost function $j\left(\left(\Omega_{i}\right),\left(C_{i}\right)\right)$ alternatively with respect to $\Omega_{i}$ and with respect to $C_{i}$. The minimization with respect to $\Omega_{i}$ consists of classifying the image, while the minimization with respect to $C_{i}$ is obtained straightforward by the mean of the image in each class:

$$
C_{i}=\frac{1}{\left|\Omega_{i}\right|} \int_{\Omega_{i}} v(x) d x \text {. }
$$

The unsupervised classification algorithm is then as follows:

- Initialization: define an initial guess $C_{1}, \ldots, C_{n}$ (e.g. equi-distributed classes).

- Repeat until convergence:

- Calculate the classified image using the classes $C_{1}, \ldots, C_{n}$ (see previous algorithm).

- Update the values of the classes using (39).

If the number $n$ of classes is not given, we can add a penalization term " $+\beta n$ " in the cost function (38), measuring the number of classes. The minimization with respect to $n$ provides the optimal number of classes. The number of classes is clearly related to the choice of the weighting coefficient $\beta$.

\section{Segmentation}

This section is concerned with image segmentation, which aim is to find a partition of an image into its constituent parts. The idea is still to apply our topological gradient based algorithm for the detection of edges in the image.

Several approaches have been studied in the literature. One can cite variational methods, for example based on the minimization of the Mumford-Shah functional [43], the active contours and snake methods [23,51], stochastic approaches $[22,24]$, wavelets, ... [9-11,44,47,48,57].

This section summarizes the main results presented in $[12,16]$. Several numerical experiments are also detailed in these references and show the efficiency of our approach.

\subsection{From restoration to segmentation}

We still consider the restoration algorithm, in which the following conductivity is used for the perturbed problem:

$$
c(\varepsilon)=\left\{\begin{array}{l}
\varepsilon \text { in } \omega, \\
\frac{1}{\varepsilon} \text { outside } \omega,
\end{array}\right.
$$

where $\omega \subset \Omega$ represents the edge set. We first assume that $\omega$ is thickened (i.e. of codimension 0 in $\Omega$ ). From equation (40), the algorithm now consists of solving the following problem:

$$
\left(\mathcal{P}_{\varepsilon}\right)\left\{\begin{array}{lll}
-\operatorname{div}\left(\varepsilon \nabla u_{\varepsilon}\right)+u_{\varepsilon}=v & \text { in } \quad \omega \\
-\operatorname{div}\left(\frac{1}{\varepsilon} \nabla u_{\varepsilon}\right)+u_{\varepsilon}=v & \text { in } \quad \Omega \backslash \omega \\
\partial_{n} u_{\varepsilon}=0 & \text { on } \quad \partial \Omega
\end{array}\right.
$$


where $u_{\varepsilon} \in H^{1}(\Omega)$, i.e. with the implicit boundary condition that $c(\varepsilon) \partial_{n} u_{\varepsilon}$ has the same value on both sides of $\partial \omega$.

Then we have the following asymptotic result [12]:

Theorem 5.1. If we denote by $u_{\varepsilon}$ the unique solution of problem $\left(\mathcal{P}_{\varepsilon}\right)$ in $H^{1}(\Omega)$, then

$$
\lim _{\varepsilon \rightarrow 0}\left(\left\|\nabla u_{\varepsilon}-\nabla u_{0}\right\|_{L^{2}(\Omega \backslash \omega)}+\left\|u_{\varepsilon}-u_{0}\right\|_{L^{2}(\omega)}\right)=0
$$

where $u_{0} \in H^{1}(\Omega \backslash \omega) \cap L^{2}(\Omega)$ is the solution to the following problem

$$
\left(\mathcal{P}_{0}\right)\left\{\begin{array}{lll}
u_{0}=v & \text { in } & \omega \\
-\operatorname{div}\left(\nabla u_{0}\right)=0 & \text { in } & \Omega \backslash \omega \\
\partial_{n} u_{0}=0 & \text { on } & \partial \omega \\
\partial_{n} u_{0}=0 & \text { on } & \partial \Omega
\end{array}\right.
$$

This result proves that the segmented image $u_{0}$ can be approximated by $u_{\varepsilon}$ if $\varepsilon$ is small. We now assume that the edge set $\omega$ is of codimension 1 in $\Omega$. From the point of view of applications, it is completely natural to assume that the edges are flat in the image. In order to have coherent notations, we will further denote by $\sigma$ the edge set. We assume that $\sigma$ is known, e.g. provided by the crack detection algorithm previously seen.

We can rewrite the approximated segmentation problem $\left(\mathcal{P}_{\varepsilon}\right)$ as follows:

$$
\left(\tilde{\mathcal{P}}_{\varepsilon}\right) \begin{cases}-\operatorname{div}\left(\frac{1}{\varepsilon} \nabla u_{\varepsilon}\right)+u_{\varepsilon}=v & \text { in } \quad \Omega \backslash \sigma \\ \partial_{n} u_{\varepsilon}=0 & \text { on } \quad \sigma \\ \partial_{n} u_{\varepsilon}=0 & \text { on } \quad \partial \Omega\end{cases}
$$

where $u_{\varepsilon} \in H^{1}(\Omega \backslash \sigma)$. If $v \in L^{2}(\Omega)$, then problem $\left(\tilde{\mathcal{P}}_{\varepsilon}\right)$ has a unique solution in $H^{1}(\Omega \backslash \sigma)$. As a corollary of the previous result, we have the following one [12]:

Theorem 5.2. If we denote by $u_{\varepsilon}$ the unique solution of problem $\left(\tilde{\mathcal{P}}_{\varepsilon}\right)$ in $H^{1}(\Omega \backslash \sigma)$, then

$$
\left\|u_{\varepsilon}\right\|_{L^{2}(\Omega)} \leq\|v\|_{L^{2}(\Omega)}, \quad\left\|\nabla u_{\varepsilon}\right\|_{L^{2}(\Omega \backslash \sigma)} \leq \sqrt{\varepsilon}\|v\|_{L^{2}(\Omega)}
$$

and

$$
\lim _{\varepsilon \rightarrow 0}\left\|\nabla u_{\varepsilon}-\nabla u_{0}\right\|_{L^{2}(\Omega \backslash \sigma)}=0
$$

where $u_{0} \in H^{1}(\Omega \backslash \sigma)$ is the unique solution to the following problem:

$$
\left(\tilde{\mathcal{P}}_{0}\right) \quad\left\{\begin{array}{lll}
-\operatorname{div}\left(\nabla u_{0}\right)=0 & \text { in } & \Omega \backslash \sigma, \\
\int_{\Omega_{i}} u_{0}=\int_{\Omega_{i}} v & & \forall \Omega_{i} \text { connex component of } \Omega \backslash \sigma, \\
\partial_{n} u_{0}=0 & \text { on } \quad \sigma, \\
\partial_{n} u_{0}=0 & \text { on } \quad \partial \Omega .
\end{array}\right.
$$

For numerical reasons, it can be very difficult to solve directly problem $\left(\tilde{\mathcal{P}}_{0}\right)$, and even problem $\left(\tilde{\mathcal{P}}_{\varepsilon}\right)$ for too small values of $\varepsilon>0$. Indeed the conditioning of the system to be solved goes to infinity when $\varepsilon \rightarrow 0$. In order to overcome this issue, we will expand the solution $u_{\varepsilon}$ of problem $\left(\tilde{\mathcal{P}}_{\varepsilon}\right)$ into a power series of $\varepsilon$.

\subsection{Power series expansion}

From the knowledge of the power series expansion of $u_{\varepsilon}$ and the computation of several solutions $u_{\varepsilon}$ for not too small coefficients $\varepsilon>0$, it is possible to approximate the asymptotic solution $u_{0}[12]$ : 
Theorem 5.3. There exist a constant $\varepsilon_{R}>0$ and a family of functions $\left(u_{n}\right)_{n \in \mathbb{N}}$ of $H^{1}(\Omega \backslash \sigma)$ such that for all $0 \leq \varepsilon \leq \varepsilon_{R}$,

$$
u_{\varepsilon}=\sum_{n=0}^{\infty} u_{n} \varepsilon^{n} .
$$

Moreover, $u_{0}$ is the unique solution in $H^{1}(\Omega \backslash \sigma)$ of problem $\left(\tilde{\mathcal{P}}_{0}\right)$, and the other functions $\left(u_{n}\right)$ are the unique solutions in $H^{1}(\Omega \backslash \sigma)$ of the following problems:

$$
\begin{aligned}
& \left(\tilde{\mathcal{P}}_{1}\right)\left\{\begin{array}{lll}
-\operatorname{div}\left(\nabla u_{1}\right)=-u_{0}+v & \text { in } \quad & \Omega \backslash \sigma, \\
\int_{\Omega_{i}} u_{1}=0 & & \forall \Omega_{i} \text { connex component of } \Omega \backslash \sigma, \\
\partial_{n} u_{1}=0 & \text { on } \quad \sigma, \\
\partial_{n} u_{1}=0 & \text { on } \quad \partial \Omega,
\end{array}\right. \\
& n \geq 2,\left(\tilde{\mathcal{P}}_{n}\right)\left\{\begin{array}{lll}
-\operatorname{div}\left(\nabla u_{n}\right)=-u_{n-1} & \text { in } & \Omega \backslash \sigma, \\
\int_{\Omega_{i}} u_{n}=0 & & \forall \Omega_{i} \text { connex component of } \Omega \backslash \sigma, \\
\partial_{n} u_{n}=0 & \text { on } \quad \sigma, \\
\partial_{n} u_{n}=0 & \text { on } \quad \partial \Omega .
\end{array}\right.
\end{aligned}
$$

We can define a function of $\varepsilon \in \mathbb{R}^{+}$as follows

$$
f(\varepsilon):=u_{\varepsilon} \in H^{1}(\Omega \backslash \sigma)
$$

From the previous theorem, we know that $f$ has a power series expansion at the origin given by (48). We consider a family of $N$ points $\left(\varepsilon_{i}\right)$ in $\left[\varepsilon_{c}, \varepsilon_{R}\right]$, where $\varepsilon_{c}$ is the smallest value of $\varepsilon$ for which it is easy to numerically compute $f(\varepsilon)$, and $\varepsilon_{R}$ is smaller than the convergence radius of the power series. We can then compute an interpolation polynomial $g_{N}$ of degree $N-1$ defined by:

$$
g_{N}(\varepsilon)=\sum_{i=1}^{N}\left(\prod_{j=1, j \neq i}^{N} \frac{\varepsilon-\varepsilon_{j}}{\varepsilon_{i}-\varepsilon_{j}}\right) u_{\varepsilon_{i}}
$$

where $N$ is the number of points $\varepsilon_{i}$.

The analycity of $f$ allows us to estimate the approximation error:

$$
\left\|u_{0}-g_{N}(0)\right\|_{H^{1}(\Omega \backslash \sigma)}=\mathcal{O}\left(\varepsilon_{c}^{N}\right) .
$$

\subsection{Algorithm}

We can then define a segmentation algorithm, based on the restoration algorithm previously defined in section 3:

- Solve the direct (21) and adjoint (27) unperturbed problems with $c=c_{0}$ everywhere.

- Compute the $2 \times 2$ matrix $M(x)$ defined by equation (28) and its lowest eigenvalue $\lambda_{\min }(M(x))$ at each point of the domain $\Omega$.

- Define $\sigma=\left\{x \in \Omega ; \lambda_{\text {min }}<\alpha<0\right\}$ the edge set, where $\alpha$ is a small negative threshold.

- Set $\varepsilon_{c}>0$ the minimal value of $\varepsilon$ for which it is easy to compute numerically the solution $u_{\varepsilon}$ of problem $\left(\tilde{\mathcal{P}}_{\varepsilon}\right)$.

- Choose $N \in \mathbb{N}^{*}$ in order to have an approximation error in $\mathcal{O}\left(\varepsilon_{c}^{N}\right)$, and choose $N$ different values $\left(\varepsilon_{i}\right)$.

- Compute the solutions $\left(u_{\varepsilon_{i}}\right)$ in $H^{1}(\Omega \backslash \sigma)$ of problems $\left(\tilde{\mathcal{P}}_{\varepsilon_{i}}\right)$.

- Compute the interpolation polynomial $g_{N}$ of degree $N-1$, defined by equation (52), for $\varepsilon=0$. 


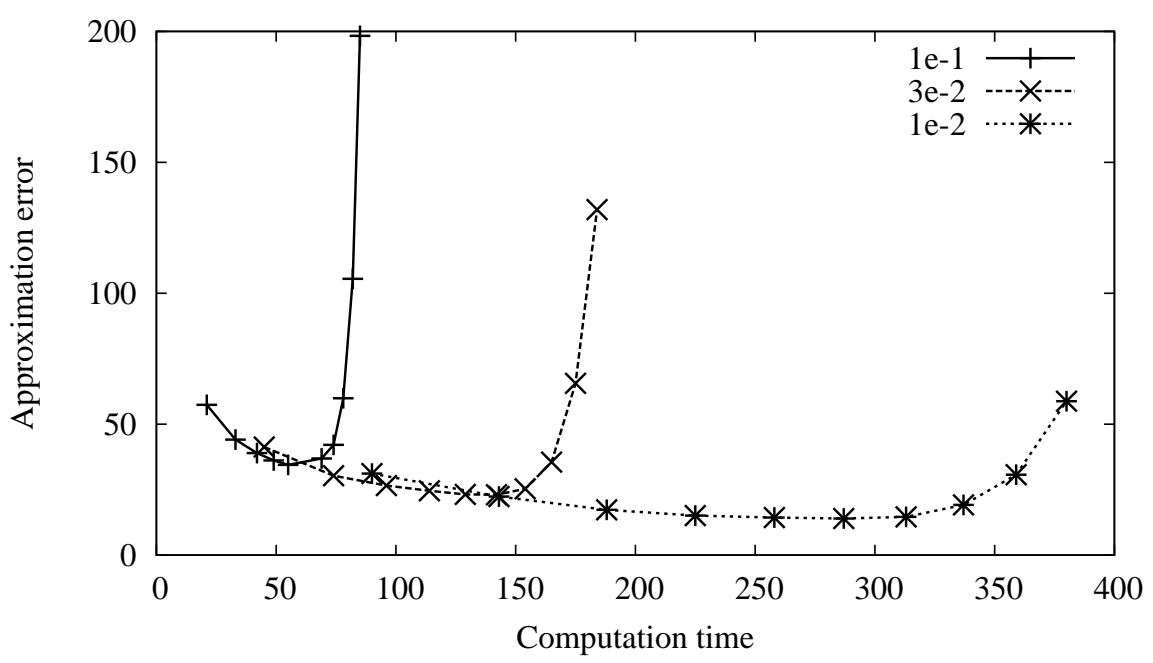

Figure 4. Segmentation: norm of the gradient of the interpolated solution on $\Omega \backslash \sigma$ for $N=$ $1 \ldots 10$ (10 points on a same curve) versus the computational cost, for several values of $\varepsilon_{c}$ : $10^{-1}, 3.10^{-2}$, and $10^{-2}$ (3 curves).

This algorithm has a complexity in $\mathcal{O}(N . n . \log (n))$, where $n$ is the number of pixels in the image, and $N$ is the degree of the interpolation approximation. In numerical experiments, $N$ is typically of the order of 2 to 5 .

Figure 4 illustrates the interest of interpolating (or extrapolating) the solution instead of considering smaller values of $\varepsilon$. We refer to [12] for more numerical results.

\section{Complexity AND SPEEDing UP}

In this section, we present the techniques that we have used for solving the PDE problems previously seen, and that lead to a theoretical complexity in $\mathcal{O}(n \cdot \log (n))$ [16]. Several numerical experiments have confirmed this complexity $[16,17]$.

\subsection{Discrete cosine transform}

In all the algorithms we presented in the previous sections, we only have to solve the following PDE

$$
\begin{cases}-\operatorname{div}(c \nabla u)+u=v & \text { in } \quad \Omega, \\ \partial_{n} u=0 & \text { on } \quad \partial \Omega\end{cases}
$$

for various coefficients $c$. The first resolutions are done with a constant value of $c$. It is then possible to largely speed up the computation time by using the discrete cosine transform (DCT) method. Problem (54) is then equivalent to

$$
\sum_{m, n}\left(1+c(m \pi)^{2}+c(n \pi)^{2}\right) u_{m, n} \phi_{m, n}=\sum_{m, n} v_{m, n} \phi_{m, n},
$$

where we denote by $\phi_{m, n}=\delta_{m, n} \cos (m \pi x) \cos (n \pi y)$ a cosine basis of $\mathbb{R}^{2}$, and where $\left(v_{m, n}\right)$ represent the DCT coefficients of the original image $v$. It is then straightforward to identify $\left(u_{m, n}\right)$, the DCT coefficients of $u$ in equation (55):

$$
u_{m, n}=\frac{v_{m, n}}{1+c(m \pi)^{2}+c(n \pi)^{2}} .
$$

The complexity of such a resolution is $\mathcal{O}(n \cdot \log (n))$, where $n$ is the number of pixels of the image. The resolution of all unperturbed problems is then done in the following way: 


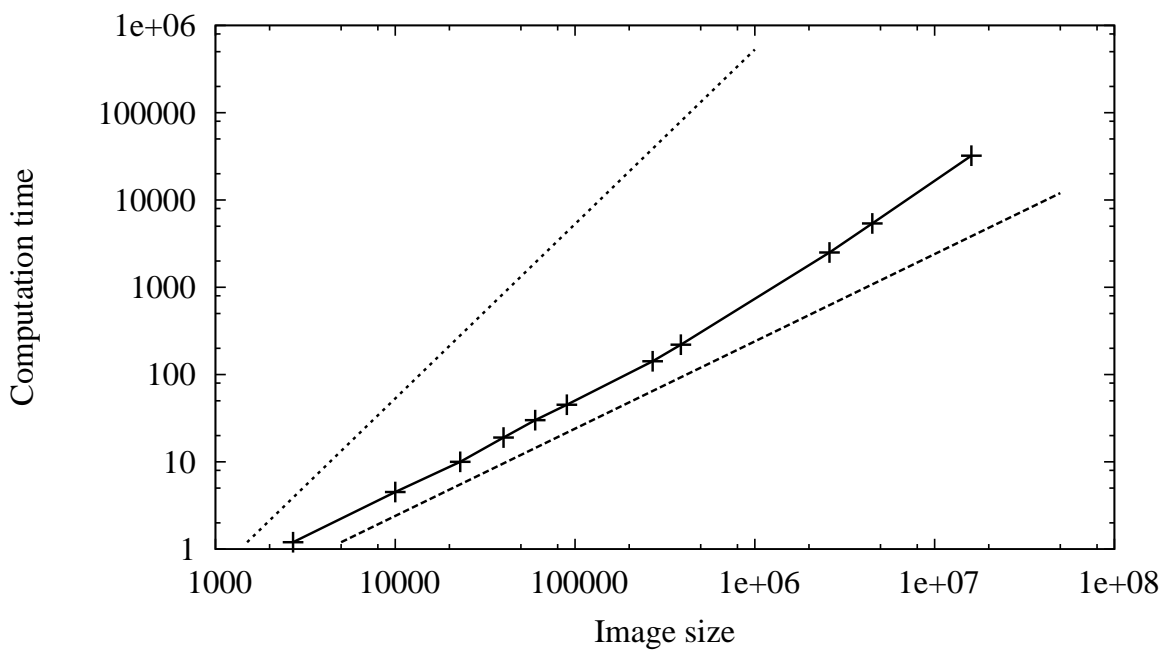

Figure 5. Computation time versus the size of the image, using a log-log scale: linear and quadratic complexity in dashed lines, result of our experiments in plain line.

- Computation of $v_{m, n}$, the DCT coefficients of the original image $v$.

- Computation of $u_{m, n}$, the DCT coefficients of $u$ from equation (56).

- Computation of $u$ using an inverse DCT.

\subsection{Preconditioned conjugate gradient}

Then, the solution of all previously detailed problems comes from the resolution of a perturbed problem. For the last resolution of a direct problem with a non constant coefficient $c$, we can rewrite the problem in the following way:

$$
A(c) u=B,
$$

where $u$ is the unknown image. If $c$ is constant, equation (57) is easy to solve. The idea is to precondition equation (57) with the DCT solver used in the first resolution. Problem (57) is equivalent to

$$
\left[A\left(c_{0}\right)^{-1} A(c)\right] u=\left[A\left(c_{0}\right)^{-1} B\right] .
$$

As $c$ is close to $c_{0}$ ( $c$ is indeed equal to $c_{0}$, except in a negligible part of the domain), the system matrix $\left[A\left(c_{0}\right)^{-1} A(c)\right]$ is close to the identity operator, and the resolution of (58) is then easy: we use a preconditioned conjugate gradient (PCG) method to solve this problem. As the coefficient $c$ is close to $c_{0}$, we can expect a $\mathcal{O}(n . \log (n))$ complexity for the resolution of the perturbed problem. The numerical experiments clearly confirm this complexity, both for small and large problems (see Figure 5).

The main advantage is that it allows us to process images in a very short time (e.g. $1600 \times 1200$ images in less than one second) and movies in real time (provided the movie is split into short sequences of a few seconds) with a $\mathrm{c}_{++}$code.

\section{Coupling Between the topological GRADient AND the Minimal Path TECHNIQUE}

As previously seen, e.g. in section 2, it is crucial to identify connected (or continuous) contours. Up to now, we had to threshold the topological gradient with a not too small value, in order to identify connected contours, but this leads to thick identified edges, and also to consider more noisy points as potential edges. 
We noticed that the edges correspond to valley lines of the topological gradient. It is of course possible to identify them by adapting the threshold coefficient, but we propose here to use the minimal path and fast marching techniques for identifying the valley lines of the topological gradient [30-32,34,46,54,60].

In the following, we consider any of the previous image processing problems. We only assume that the topological gradient $g$ has been defined and computed everywhere. The goal is to identify the valley lines corresponding to the most negative parts of the topological gradient.

This section summarizes the study presented in [1], in which several numerical experiments are shown in the case of segmentation and inpainting.

\subsection{Minimal paths}

Let $g$ be the topological gradient. The idea of the minimal path technique is to define a potential function, measuring in some sense for any point of $\Omega$ the cost for a path to contain this point. As we want to identify paths in the most negative part of the topological gradient, and considering that the potential function must be positive, we define the following function:

$$
P(x)=g(x)-\min _{y \in \Omega}\{g(y)\} .
$$

We simply shift the topological gradient from its minimal value, in order to obtain a positive function. We can see that the points where the topological gradient $g$ reaches its minimal value are costless. This is a way to consider that these points must be on the minimal paths.

We denote by $C(s)$ a path (or curve) in the image, where $s$ represents the curvilign coordinate. We can now define a cost function, measuring the cost of such a path:

$$
J(C)=\int_{C}(P(C(s))+\alpha) d s,
$$

where $\alpha>0$ is a positive regularization coefficient, measuring the length of this path.

The goal is to minimize $J$, in order to find the shortest and least costly path between two points. For this purpose, we define the following distance function:

$$
D\left(x ; x_{0}\right)=\inf _{C \in \mathcal{A}\left(x, x_{0}\right)} J(C),
$$

where $\mathcal{A}\left(x, x_{0}\right)$ is the set of all paths going from $x_{0}$ to $x$ in the image.

\subsection{Fast marching}

The fastest way to compute the distance function defined by equation (61) is to solve a front propagation equation:

$$
\frac{\partial C(s, t)}{\partial t}=\frac{1}{P(C(s, t))+\alpha} \mathbf{n}_{C}(s, t),
$$

where $\mathbf{n}_{C}(s, t)$ is the outer normal unit vector to the front $C$. We initialize the propagation with $C(s, 0)$ equal to a infinitely small circle centered at $x_{0}$.

This path evolves with a propagation speed inversely proportional to the potential function. If for example a point in the outer part of the front has a large potential (i.e. a large cost), then the propagation speed will be nearly equal to 0 and the front will not expand at this point. From the theory of Eikonal equations, the distance $D\left(x ; x_{0}\right)$ is simply the instant $t$ at which the front, initialized at point $x_{0}$, reaches point $x[34,60]$. 


\subsection{Coupled algorithm}

We can certainly consider that the global minimum of the topological gradient is part of the edge set. So we can choose the reference point $x_{0}$ as being this minimum. It is then possible to compute the distance between $x_{0}$ and any other point $x$. A gradient descent algorithm can then be used to minimize the distance function, in order to find the minimal path between $x_{0}$ and the initial point of the optimization scheme. If these two points are part of the same edge, as the potential function has been defined such that it is relatively costless to remain on the edge (thanks to the topological gradient), the minimal path will be a very good approximation of this edge. The main advantage is now that we are sure that this path corresponds to a continuous contour.

For a small additional computation cost, it is possible to consider more than one reference point. The distance function corresponds then to the distance to the set of these points. The corresponding Voronoï diagram can be seen as a dual mesh, and the minimum of the distance function on each edge of this mesh is a saddle-point: minimal distance along the edges of the mesh, maximal distance to the reference points.

The hybrid algorithm we propose is the following:

- Compute the topological gradient of the image (see previous sections).

- Choose $N$ key-points; the main one will be for example the global minimum of the topological gradient.

- Fast marching: computation of the distance function to all these key-points, and of the corresponding Voronoï diagram.

- Saddle-points: on each edge of the Voronoï diagram, determine the point of minimal distance.

- Sort all these saddle-points, from smaller to larger distance.

- For each of these points, from smaller to larger distance, check if it will not be used for connecting two key-points, one of which is already connected to two other key-points.

- If this is not the case, use this point as an initialization for a descent type algorithm in order to connect the two corresponding key-points.

This algorithm clearly converges, and all the key-points are connected to at most two other key-points at convergence. This provides a continuous contour, connecting the key-points. It is then an approximation of one of the main contours of the image as it corresponds to a valley line of the topological gradient.

As seen in [1], it allows us to appreciably improve our inpainting algorithm. It also improves the quality of the segmentation (see e.g. Figure 6). For all other image processing problems, there were no noticeable improvements.

\section{Conclusions and Perspectives}

We presented in this article many applications in image processing of the crack detection technique, based on the topological gradient. It provides an excellent frame for solving all these image processing problems. It has been successfully applied to image inpainting, restoration, classification and segmentation. In all these cases, we obtain excellent results and the computing time is very small.

We have also seen that this technique can be applied to color images as well as grey-level images, but also three-dimensional images, or movies, without any trouble. The theoretical complexity, confirmed by numerical experiments, allows us to process movies in nearly real time (on a dual-processor laptop, with a c++ code).

Another interesting point is that all these algorithms rely on the same kernel, as we always solve the same kind of PDE problems. This makes the implementation much more easy.

Several perspectives are currently under study. We can cite here the possibility of taking into account higher order differential operators, with the aim of a better reconstruction of the gradient of the image. For instance, in the case of inpainting problems, the inpainted image is piecewise affine. With the same kind of approach, we should be able to reconstruct more precisely the gradient of the image, and then the image itself. 

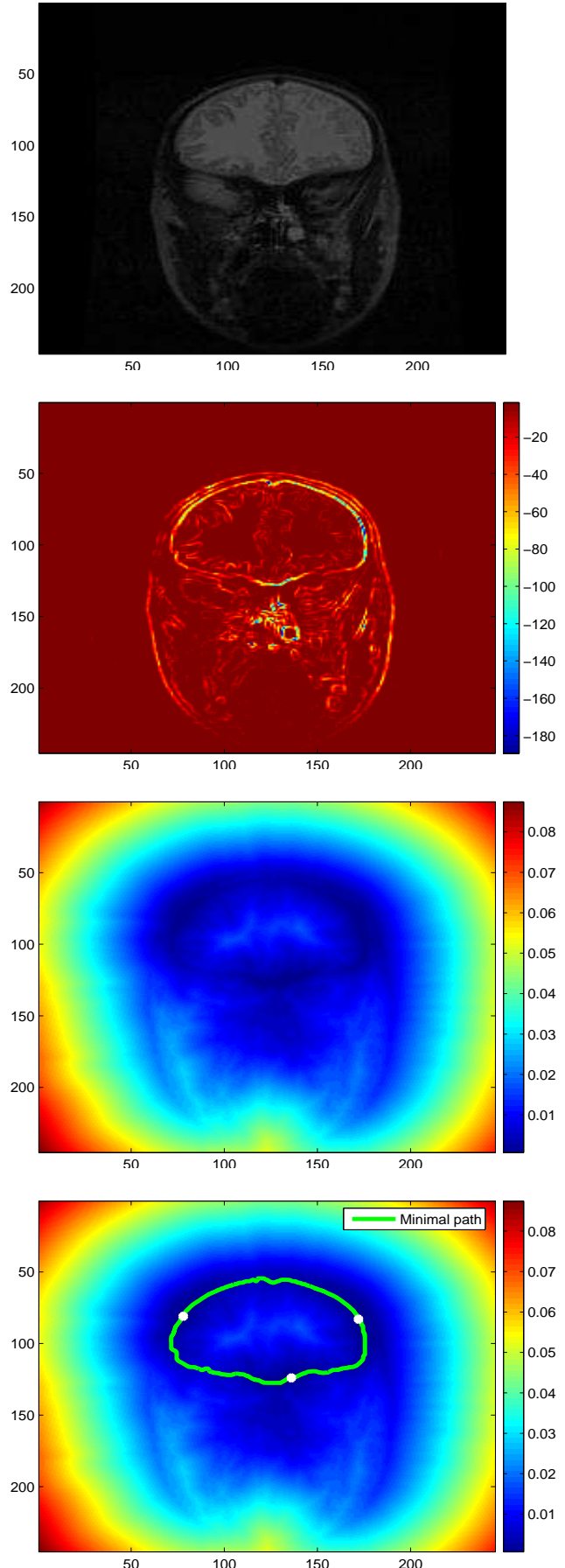
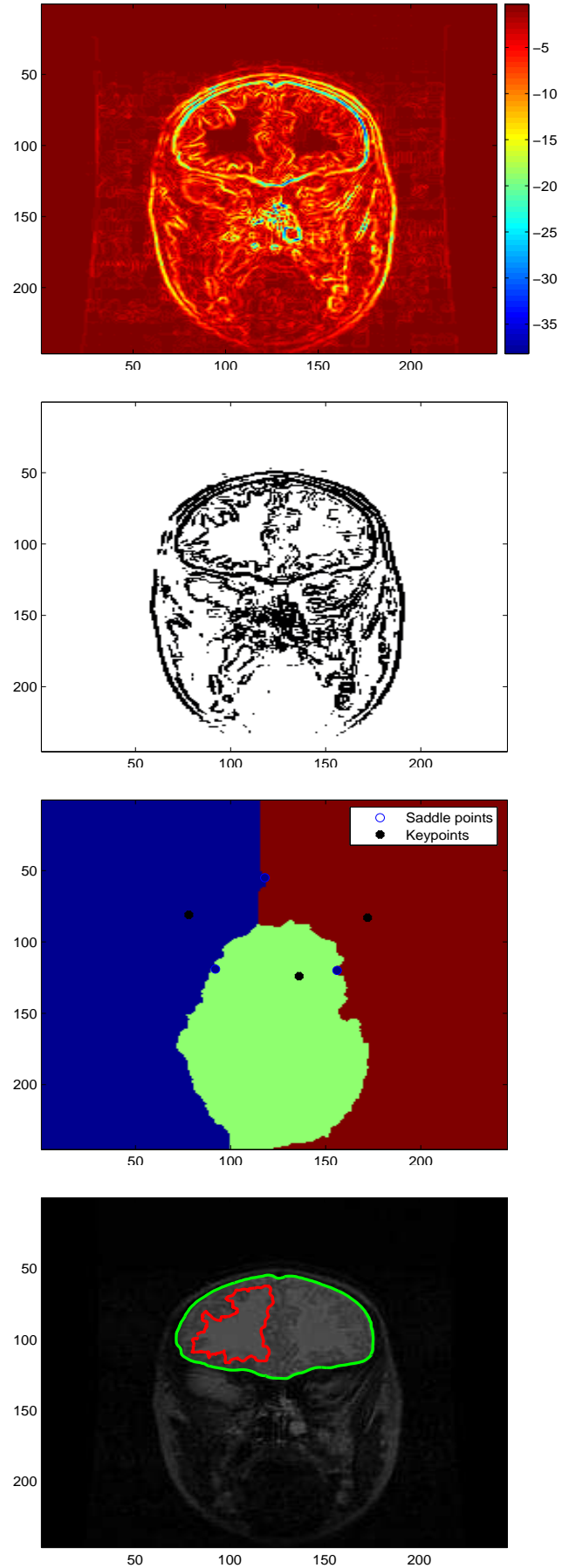

Figure 6. From top to bottom: Original image (left); Standard gradient (right). Topological gradient (left); Identified edges by the topological gradient (right). Distance function to 3 selected key-points (left); Voronoï diagram (right). Minimal path between the key-points (left); Two identified continuous contours of the original image (right). 


\section{REFERENCES}

[1] Y. Ahipo, D. Auroux, L. Cohen, and M. Masmoudi. A hybrid scheme for contour detection and completion based on topological gradient and fast marching algorithms - an application to image inpainting and segmentation. Preprint, 2008.

[2] G. Alessandrini, E. Beretta, and S. Vessela. Determining linear cracks by boundary measurements: Lipschitz stability. SIAM J. Math. Anal., 27(2):361-375, 1996.

[3] G. Alessandrini and A. Diaz Valenzuela. Unique determination of multiple cracks by two measurements. SIAM J. Control Optim., 34(3):913-921, 1996.

[4] G. Allaire, F. Jouve, and A. M. Taoder. A level set method for shape optimization. C. R. Acad. Sci. Paris Ser. I, 334:1125-1130, 2002.

[5] G. Allaire and R. Kohn. Optimal design for minimum weight and compliance in plane stress using extremal microstructures. Eur. J. Mech. A Solids, 12:839-878, 1993.

[6] H. Ammari, M. S. Vogelius, and D. Volkov. Asymptotic formulas for perturbations in the electromagnetic fields due to the presence of inhomogeneities of small diameter ii - the full maxwell equations. J. Math. Pures Appl., 80(8):769-814, 2001.

[7] S. Amstutz, I. Horchani, and M. Masmoudi. Crack detection by the topological gradient method. Control and Cybernetics, 34(1):119-138, 2005.

[8] S. Andrieux and A. Ben Abda. Identification of planar cracks by complete overdetermined data: inversion formulae. Inverse Problems, 12:553-563, 1996.

[9] G. Aubert and J.-F. Aujol. Optimal partitions, regularized solutions, and application to image classification. Applicable Analysis, 84(1):15-35, 2005.

[10] G. Aubert and P. Kornprobst. Mathematical Problems in Image Processing, volume 147 of Applied Mathematical Sciences. Springer-Verlag, 2001.

[11] J.-F. Aujol, G. Aubert, and L. Blanc-Féraud. Wavelet-based level set evolution for classification of textured images. IEEE Trans. Image Proc., 12(12):1634-1641, 2003.

[12] D. Auroux. From restoration by topological gradient to medical image segmentation via an asymptotic expansion. Math. Comput. Model., 2008. Accepted for publication.

[13] D. Auroux, L. Jaafar Belaid, and M. Masmoudi. A topological asymptotic analysis for the regularized grey-level image classification problem. Math. Model. Numer. Anal., 41(3):607-625, 2007.

[14] D. Auroux, L. Jaafar Belaid, and B. Rjaibi. Application of the topological gradient method to color image restoration. Preprint, 2008.

[15] D. Auroux and M. Masmoudi. A one-shot inpainting algorithm based on the topological asymptotic analysis. Comp. Appl. Math., 25(2-3):1-17, 2006.

[16] D. Auroux and M. Masmoudi. Image processing by topological asymptotic expansion. J. Math. Imaging Vision, 2008. Accepted for publication.

[17] D. Auroux, M. Masmoudi, and L. Jaafar Belaid. Image restoration and classification by topological asymptotic expansion. Variational Formulations in Mechanics: Theory and Applications, E. Taroco, E.A. de Souza Neto and A.A. Novotny (Eds). CIMNE, Barcelona, Spain, 2006.

[18] Z. Belhachmi and D. Bucur. Stability and uniqueness for the crack identification problem. SIAM J. Control Optim., 46(1):253$263,2007$.

[19] A. Ben Abda, H. Ben Ameur, and M. Jaoua. Identification of 2d cracks by elastic boundary measurements. Inverse Problems, 15:67-77, 1999.

[20] A. Ben Abda, M. Kallel, J. Leblond, and J.-P. Marmorat. Line-segment cracks recovery from incomplete boundary data. Inverse Problems, 18:1057-1077, 2002.

[21] M. Bendsoe. Optimal topology design of continuum structure: an introduction. Technical report, Department of Mathematics, Technical University of Denmark, Lyngby, Denmark, 1996.

[22] M. Berthod, Z. Kato, S. Yu, and J. Zerubia. Bayesian image classification using markov random fields. Image Vision Comput., 14(4):285-293, 1996.

[23] A. Blake and M. Isard. Active Contours. Springer-Verlag, 1998.

[24] C. A. Bouman and M. Shapiro. A multiscale random field model for bayesian image segmentation. IEEE Trans. Image Proc., 3:162-177, 1994.

[25] K. Bryan and M. S. Vogelius. A review of selected works on crack identification. In Proc. IMA workshop on Geometric Methods in Inverse Problems and PDE Control, August 2001.

[26] A.-P. Calderón. On an inverse boundary value problem. In Seminar on Numerical Analysis and Its Applications to Continuum Physics, pages 65-73, Rio de Janeiro, Brasil, 1980. Soc. Brasil. Mat.

[27] F. Catté, T. Coll, P.-L. Lions, and J.-M. Morel. Image selective smoothing and edge detection by non linear diffusion. SIAM J. Numer. Anal., 29:182-193, 1992.

[28] T. Chan and J. Shen. Mathematical models for local deterministic inpaintings. Technical Report 00-11, UCLA CAM, 2000.

[29] T. Chan and J. Shen. Non-texture inpainting by curvature-driven diffusions (ccd). Technical Report 00-35, UCLA CAM, 2000. 
[30] L. D. Cohen. Multiple contour findind and perceptual grouping using minimal paths. Technical report, Les Cahiers du CEREMADE, Université Paris Dauphine, 2001.

[31] L. D. Cohen and R. Kimmel. Global minimum for active contour models: a minimal path approach. Int. J. Computer Vision, 24(1):57-78, 1997.

[32] T. Deschamps and L. D. Cohen. Minimal path in 3d images and application to virdual endoscopy. Technical report, Les Cahiers du CEREMADE, Université Paris Dauphine, 2000.

[33] S. Di Zenzo. A note on the gradient of a multi-image. Comput. Vision Graph. Image Proc., 33:116-125, 1986.

[34] J. Dicker. Fast marching methods and level set methods: an implementation. PhD thesis, University of British Columbia, 2006.

[35] M. Elad and M. Aharon. Image denoising via sparse and redundant representations over learned dictionaries. IEEE Trans. Image Proc., 15(12):3736-3745, 2006.

[36] M. Elad, J.-L. Starck, P. Querre, and D. L. Donoho. Simultaneous cartoon and texture image inpainting using morphological component analysis (mca). J. Appl. Comput. Harmonic Anal., 19:340-358, 2005.

[37] A. Friedman and M. S. Vogelius. Determining cracks by boundary measurements. Indiana Univ. Math. J., 38(3):527-556, 1989.

[38] S. Garreau, P. Guillaume, and M. Masmoudi. The topological asymptotic for pde systems: The elasticity case. SIAM J. Control Optim., 39(6):1756-1778, 2001.

[39] P. Guillaume and K. Sididris. The topological asymptotic expansion for the dirichlet problem. SIAM J. Control Optim., 41(4):1042-1072, 2002.

[40] R. Kohn and M. Vogelius. Relaxation of a variational method for impedance computed tomography. Comm. Pure Appl. Math., 40(6):745-777, 1987.

[41] Y. Li and F. Santosa. A computational algorithm for minimizing total variation in image restoration. IEEE Trans. Image Proc., 5:987-995, 1996.

[42] M. Masmoudi. The topological asymptotic, volume 16 of Computational Methods for Control Applications, $R$. Glowinski, H. Karawada, and J. Périaux (Eds.), pages 53-72. GAKUTO Internat. Ser. Math. Sci. Appl., Tokyo, Japan, 2001.

[43] D. Mumford and J. Shah. Optimal approximations by piecewise smooth functions and associated variational problems. Comm. Pure Appl. Math., 42(5):577-685, 1989.

[44] N. Paragios and R. Deriche. Geodesic active regions and level set methods for supervised texture segmentation. Int. J. Comput. Vision, 46(3):223-247, 2002.

[45] P. Perona and J. Malik. Scale space and edge detection detection using anisotropic diffusion. IEEE Trans. Pattern Anal. Mach. Intel., 12:629-639, 1990.

[46] N. Rawlinson and M. Sambridge. The fast marching method: an effective tool for tomographic imaging and tracking multiple phases in complex layered media. Explor. Geophys., 36:341-350, 2005.

[47] C. Samson, L. Blanc-Féraud, G. Aubert, and J. Zerubia. A level set model for image classification. Int. J. Comput. Vision, 40(3):187-197, 2000.

[48] C. Samson, L. Blanc-Féraud, G. Aubert, and J. Zerubia. A variational model for image classification and restoration. IEEE Trans. Pattern Anal. Mach. Intel., 22(5):460-472, 2000.

[49] F. Santosa. A level-set approach for inverse problems involving obstacles. ESAIM Control Optim. Calc. Var., 1:17-33, 1996.

[50] F. Santosa and M. Vogelius. A computational algorithm to determine cracks from electrostatic boundary measurements. Int. J. Eng. Sci., 29:917-937, 1991.

[51] J. A. Sethian. Level Set Methods and Fast Marching Methods: Evolving Interfaces in Computational Geometry, Fluid Mechanics, Computer Vision, and Materials Sciences. Cambridge Monograph on Applied and Computational Mathematics. Cambridge University Press, Cambridge, 1999.

[52] J. Sokolowski and A. Zochowski. On the topological derivative in shape optimization. SIAM J. Control Optim., 37:1241-1272, 1999.

[53] J. Sokolowski and A. Zochowski. Topological derivatives of shape functionals for elasticity systems. Int. Ser. Numer. Math, 139:231-244, 2002.

[54] A. Telea and J. van Wijk. An augmented fast marching method for computing skeletons and centerlines. In Proc. Eurographics IEEE-TCVG Symposium on Visualization, Vienna, 2002. Springer.

[55] J. Weickert. Theoretical foundations of anisotropic diffusion in image processing. In Theoretical Foundations of Computer Vision, volume 11, pages 221-236, Dagstuhl, Germany, 1994.

[56] J. Weickert. Anisotropic diffusion in image processing. PhD thesis, University of Kaiserslautern, Germany, 1996.

[57] J. Weickert. Efficient image segmentation using partial differential equations and morphology. Pattern Recognition, 34(9):1813$1824,2001$.

[58] P. Wen, X. Wu, and C. Wu. An interactive image inpainting method based on rbf networks. In Third International Symposium on Neural Networks, pages 629-637, 2006.

[59] T. Zhou, F. Tang, J. Wang, Z. Wang, and Q. Peng. Digital image inpainting with radial basis functions. J. Image Graphics (In Chinese), 9(10):1190-1196, 2004.

[60] F. Zhu and J. Tian. Modified fast marching methods and level set method for medical image segmentation. J. X-ray Sci. Tech, 11(4):193-204, 2003. 